\title{
ANEURISMA DA VEIA FEMORAL SIMULANDO UMA HÉRNIA INGUINAL
}

\author{
Femoral vein aneurysm simulating an inguinal hernia \\ Victor Assad BUFFARA-JR, Júlio Cezar. Uili COELHO, Mateus Martinelli de OLIVEIRA
}

ABCDDV/679

Buffara Jr. VA, Coelho JCU, Oliveira MM. Aneurisma da veia femoral simulando uma hérnia inguinal. ABCD Arq Bras Cir Dig 2009;22(4):233-4 RESUMO - Introdução - Somente seis casos de aneurismas da veia femoral simulando hérnia inguinal foram descritos na literatura. Relato do caso - Um caso de aneurisma da veia femoral comum direita que simulava uma hérnia inguinal é descrito em jovem de 19 anos de idade com uma massa dolorosa de consistência mole na região inguinal direita de seis meses de duração. Durante a consulta médica, o paciente morreu durante em episódio de convulsão generalizada. Na necropsia, tromboembolismo pulmonar maciço e um aneurisma da veia femoral comum de 8x8x7 cm com trombos foram diagnosticados.

DESCRITORES - Aneurisma. Tromboembolismo. Embolia pulmonar. Trombose.

\section{INTRODUÇÃO}

A primeira descrição de aneurisma venoso foi realizada por Osler em 1913, o qual relatou um aneurisma artério-venoso dos vasos axilares². Posteriormente, aneurismas venosos foram descritos em veias de várias outras regiões anatômicas ${ }^{1,5,6,7}$. Somente seis casos de aneurismas da veia femoral simulando hérnia inguinal foram descritos na literatura ${ }^{1,2}$. O objetivo deste trabalho é apresentar o caso de um jovem que morreu devido à tromboembolismo pulmonar maciço secundário a um aneurisma da veia femoral com trombos.

\section{RELATO DO CASO}

Paciente do sexo masculino, 19 anos, apresentou-se no consultório médico com queixa de uma massa dolorosa na região da virilha direita com seis meses de duração. Relatou que tinha consultado outro cirurgião no dia anterior, que o tinha examinado e estabelecido o diagnóstico de hérnia inguinal encarcerada.

Ao deitar na mesa de exame, apresentou crise convulsiva generalizada, vômitos, cianose e morreu em poucos minutos, apesar da tentativa de ressuscitação cardiorrespiratória. A família relatava que o paciente nunca teve crises convulsivas, dor torácica ou dificuldade respiratória anteriores. O corpo foi encaminhado ao Instituto

Trabalho realizado na Disciplina de Cirurgia do Aparelho Digestivo da Universidade Federal do Paraná, Curitiba, PR, Brasil

Endereço para correspondência: Júlio Coelho, e-mail: coelhojcu@yahoo.com.br
Médico Legal para necropsia. As artérias pulmonares de grande, médio e pequeno calibre apresentavam-se totalmente ocluídas por trombos. Na região inguinal direita, evidenciou-se aneurisma da veia femoral comum de $8 \times 8 \times 7 \mathrm{~cm}$ preenchido por sangue parcialmente coagulado e trombos aderidos à parede. $\mathrm{O}$ diagnóstico da causa da morte foi de tromboembolismo pulmonar maciço. Como diagnóstico secundário foi observado edema cerebral, possivelmente devido à anóxia cerebral.

\section{DISCUSSÃO}

Aneurismas venosos verdadeiros ou primários são dilatações saculares das veias, que contém todas as três camadas da parede da veia (íntima, média e adventícia) ${ }^{2}$. Estes aneurismas são muito raros e podem ocorrer em qualquer idade e apresentam distribuição similar em ambos os $\operatorname{sexos}^{1,5}$. Eles podem ocorrer em qualquer veia, inclusive na veia femoral comum ${ }^{3.4}$. Quando nela localizados, podem simular hérnia inguinal. Existem apenas seis desses casos descritos na literatura1. Este paciente apresentava uma massa inguinal, que também foi interpretada inicialmente como hérnia inguinal.

Esses aneurismas frequentemente apresentam-se como uma massa subcutânea macia e compressível com a palpação ${ }^{2}$. Devido a sua raridade, o diagnóstico pré-operatório é muito difícil $1,2,3,4,5$. A venografia, a ultra-sonografia duplex, a angiotomografia e a angiorressonância podem estabelecer o diagnóstico ${ }^{2,4}$. Quando o diagnóstico não é suspeitado no pré-operatório e é estabelecido durante uma operação programada de her- 
niorrafia, o cirurgião deve interromper o procedimento e posteriormente programar o tratamento definitivo ${ }^{1}$.

Além da dificuldade de estabelecer o correto diagnóstico, os aneurismas venosos podem estar associados a complicações graves, que incluem trombose, embolia e rotura $^{2,5}$. A embolia pulmonar pode ser pequena e recorrente ou ser maciça e fatal, como neste paciente. A necropsia do caso em questão evidenciou que a causa da sua morte foi embolia pulmonar maciça, cuja fonte dos êmbolos foi um aneurisma da veia femoral com trombos no seu interior. O edema cerebral observado na necropsia e a convulsão generalizada foram possivelmente secundários à anóxia causada pela embolia pulmonar maciça.

Buffara Jr. VA, Coelho JCU, Oliveira MM. Femoral vein aneurysm simulating an inguinal hernia. ABCD Arq Bras Cir Dig 2009;22(4):233-4

ABSTRACT - Background - Only six cases of femoral vein aneurysm are related on medical literature. Case report - A case of a right common femoral vein aneurysm simulating an inguinal hernia in a 19 year-old male. He had a soft and painful mass in the right inguinal area of six months of duration. At medical consultation, the patient died during a generalized convulsive episode. At necropsy, massive pulmonary thromboembolism and an 8x8 $7 \mathrm{~cm}$ common femoral vein aneurysm with thrombus were recognized.

HEADINGS: Aneurysm. Tromboembolism. Pulmonary embolia. Thrombosis.

\section{REFERÊNCIAS}

1. Castaldo ET, Williams EH, Jeffrey D, Mark P, Thomas N, Guzman RJ. Common femoral vein aneurysm simulating an inguinal hernia. Am Surg 2005;71:591-4.

2. Castle JM, Arous EJ. Femoral vein aneurysm: a case report and review of the literatura. Cardiovasc Surg 1995;3:317-9.

3. Faraj W, Selmo F, Hindi M, Haddad F, Khalil I. Cephalic vein aneurysm. Ann Vasc Surg. 2007;21:804-6.

4. Friedman SGH, Krishnasastry KV, Doscher W. Primary venous aneurysms. Surgery 1990;108:92-5.
5. Hurwitz RL, Gelabert H. Thrombosed iliac venous aneurysm: a rare cause of left lower extremity venous obstruction. J Vasc Surg 1989; 9:822-4.

6. Lorenzato MM, Granzotto E, Barros ADB, Silveira NZA, Souza PHR. Aneurisma venoso na junção esplenomesentérica e emergência da veia porta: relato de caso. Radiol Bras 2009;42:199-201.

7. Thomazinho F, Diniz JAM, El Hosni Junior RA, Diniz CAM, Perozin IS. Aneurisma da veia poplítea: relato de caso e revisão de literatura. J Vasc Bras 2008;7:262-5.

Fonte de financiamento: não há Conflito de interesse: não há Recebido para publicação:19/04/2008 Aceito para publicação: 14/10/2008 